\title{
Analytical STEM of Amorphous and Crystalline Mineral Phases in Calcium Bodies of Terrestrial Crustaceans
}

\author{
Miloš Vittori ${ }^{1}$, Vesna Srot $^{2}$, Birgit Bussmann ${ }^{2}$, Peter A. van Aken ${ }^{2}$ and Jasna Štrus ${ }^{1}$ \\ ${ }^{1 .}$ Department of Biology, Biotechnical Faculty, University of Ljubljana, Večna pot 111, Ljubljana, \\ Slovenia. \\ 2. Stuttgart Center for Electron Microscopy, Max Planck Institute for Solid State Research, \\ Heisenbergstrasse 1, 70569 Stuttgart, Germany.
}

Terrestrial isopod crustaceans (woodlice) from the family Trichoniscidae possess calcium bodies, specialized bacteria-harboring organs that accumulate calcium minerals. Layers of mineralized extracellular matrix are periodically deposited and resorbed in these organs during the moult cycle and likely function as reservoirs for calcium salts, which are an important component of the exoskeleton [1]. The cave woodlouse Titanethes albus possess two pairs of calcium bodies, both of which are filled with bacteria. By contrast, two different pairs of calcium bodies are present in the woodlouse Hyloniscus riparius. The posterior pair is filled with bacteria, whereas the anterior pair lacks bacteria [2]. Bacteriacontaining calcium bodies continuously accumulate calcium phosphate. During preparation for moult, an additional layer of glassy matrix forms around the bacteria-containing central matrix. This layer contains less phosphorus than the mineral in the central matrix and is resorbed after moult. The bacteriafree calcium bodies, on the other hand, accumulate only calcium carbonate.

In the present study we analysed the mineral deposits in the two pairs of calcium bodies at high spatial and high energy resolution using analytical scanning transmission electron microscopy (STEM), which enabled us to determine the fine-scale structure and composition of the mineral deposits in calcium bodies of $T$. albus and $H$. riparius. Isolated calcium body matrices were dehydrated in methanol and embedded in Spurr's resin. In order to avoid potential loss or recrystallization of highly soluble minerals, ultrathin sections were prepared without floating them on water. The mineralised matrices were imaged and analysed with the Zeiss SESAM and JEOL ARM200F microscopes. Energy-dispersive X-ray spectroscopy (EDX) and electron energy-loss spectroscopy (EELS) were used to characterize the Ca-containing minerals. Selected area electron diffraction (SAED) experiments were performed with a Zeiss 912 Omega transmission electron microscope with an in-column omega energy filter.

As demonstrated with EDX and SAED, the bacteria-filled matrix of the posterior calcium bodies is continuously mineralised with calcium phosphate in the form of apatite crystals a few hundred nanometres in size. As we were able to demonstrate with a combination of high resolution analytical STEM and SAED, apatite and calcite are present as distinct phases in the bacteria-filled mineralised matrix in $T$. albus but not in $H$. riparius. The transient glassy layer that is deposited between the bacteria and calcium body epithelium during preparation for moult consists is mineralised with a mixture of amorphous calcium carbonate and amorphous calcium phosphate in both species. The mineralised matrix in the anterior calcium bodies of $H$. riparius, which lack bacteria, consists entirely of calcium carbonate without apparent amounts of phosphorus. As demonstrated with SAED and EELS, the matrix in the anterior calcium bodies is mineralised with amorphous calcium carbonate.

As the glassy matrix layer of the posterior calcium bodies and the mineralised matrix of the anterior 
calcium bodies are transient and are completely resorbed after moult, their mineralisation with amorphous calcium minerals is likely biologically functional due to their high solubility under physiological conditions. Previous results indicating the presence of calcite in the anterior calcium bodies [2] are likely the consequence of thermal instability of the amorphous calcium carbonate in these structures. Future studies focusing on the mechanisms of mineral deposition and resorption in calcium bodies will contribute to the field of biomineralisation and may result in biomedical applications. Our results indicate that the presence of bacteria may be essential for the turnover of calcium phosphate in calcium bodies. Further studies of this unique relationship between bacteria and animals in a biomineralisation process may shed light on the role of bacteria in pathological mineralisation processes in humans.

[1] M Vittori, N Žnidaršič, K Žagar, M Čeh, and J Štrus. Journal of Structural Biology 180 (2012), pp. 216-225.

[2] M Vittori, A Rozman, J Grdadolnik, U Novak, and J Štrus. PLoS One 8 (2013), e58968.

[3] The research leading to these results has received funding from the European Union Seventh Framework Program [FP/2007-2013] under grant agreement No.312483 (ESTEEM2) and the Slovenian Research Agency, research program P1-0184.
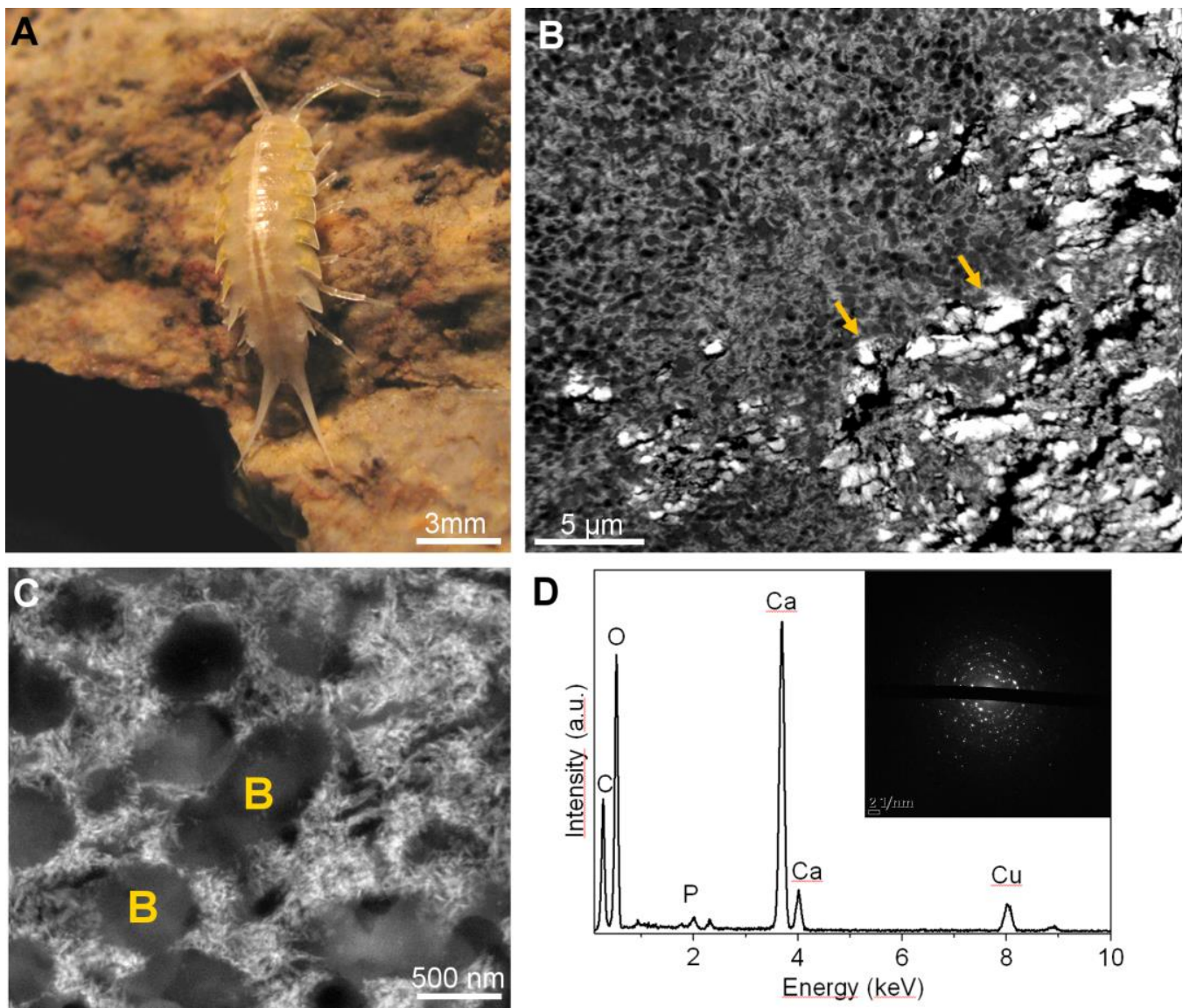

Figure 1. A: The cave woodlouse, Titanethes albus. B: HAADF-STEM image of the bacteriacontaining matrix with distinct regions mineralised with either calcite (arrows) or apatite. C: Higher magnification HAADF-STEM image showing apatite crystals surrounding bacterial casts (B). D: EDX spectrum and SAED (inset) of a calcite containing region of the matrix. 